Review

\title{
Dual Functions of Natural Killer Cells in Selection and Differentiation of Stem Cells; Role in Regulation of In- flammation and Regeneration of Tissues
}

\author{
Anahid Jewett ${ }^{\circledR}$, Yan-Gao Man², Han-Ching Tseng' \\ 1. The Jane and Jerry Weintraub Center for Reconstructive Biotechnology, and Division of Oral Biology and Medicine, UCLA School of \\ Dentistry, University of California, Los Angeles, CA 90095; \\ 2. The Diagnostic and Translational Research Center, Henry Jackson Foundation, Gaithersburg, MD.
}

$\triangle$ Corresponding author: Division of Oral Biology and Medicine, UCLA School of Dentistry, 10833 Le Conte Ave. Los Angeles, CA. 90095-1668 Telephone: (310) 206-3970 Fax: (310) 794-7109 E-mail: ajewett@ucla.edu.

(C) Ivyspring International Publisher. This is an open-access article distributed under the terms of the Creative Commons License (http://creativecommons.org/ licenses/by-nc-nd/3.0/). Reproduction is permitted for personal, noncommercial use, provided that the article is in whole, unmodified, and properly cited.

Received: 2012.11.11; Accepted: 2012.11.25; Published: 2012.12.01

\begin{abstract}
Accumulated evidence from our laboratory indicates that conditioned or anergized NK cells have the ability to induce resistance of healthy stem cells and transformed cancer stem cells through both secreted factors and direct cell-cell contact by inducing differentiation. Cytotoxic function of NK cells is suppressed in the tumor microenvironment by a number of distinct effectors and their secreted factors. Furthermore, decreased peripheral blood NK cell function has been documented in many cancer patients. We have previously shown that NK cells mediate significant cytotoxicity against primary oral squamous carcinoma stem cells (OSCSCs) as compared to their more differentiated oral squamous carcinoma cells (OSCCs). In addition, human embryonic stem cells (hESCs), human mesenchymal stem cells (hMSCs), human dental pulp stem cells (hDPSCs) and induced human pluripotent stem cells (hiPSCs) were all significantly more susceptible to NK cell mediated cytotoxicity than their differentiated counterparts or parental cells from which they were derived. We have also reported that inhibition of differentiation or reversion of cells to a less-differentiated phenotype by blocking NFKB or gene deletion of COX2 significantly augmented NK cell function. Furthermore, the induction of resistance of the stem cells to NK cell mediated cytotoxicity and their subsequent differentiation is amplified when either the stem cells or the NK cells were cultured in the presence of monocytes. Therefore, we propose that the two stages of NK cell maturation namely CD 16+CD56dimCD69- NK cells are important for the lysis of stem cells or poorly differentiated cells whereas the CDI6dim/-CD56dim/+CD69+NK cells are important for differentiation and eventual regeneration of the tissues and the resolution of inflammation, thus functionally serving as regulatory $N K$ cells $\left(N K_{\text {reg }}\right)$. CDI 6 receptor on the NK cells were found to be the receptor with significant potential to induce NK cell anergy, however, our recent data indicated that NKp46 but not NKp30 or NKp44 were also able to induce significant anergy in NK cells, although the levels were less when compared to CDI6 receptor triggering. The concept of split anergy in NK cells and generation of $\mathrm{NK}_{\text {reg }}$ and its contribution to cell differentiation, tissue repair and regeneration and in tumor resistance will be discussed in this review.
\end{abstract}

Key words: apoptosis, NFKB, NK, cancer stem cells, differentiation, Regulation.

\section{Introduction}

NK cells are known to mediate direct cytotoxicity as well as antibody-dependent cellular cytotoxicity (ADCC) against a variety of tumor cells. By pro- ducing key cytokines and chemokines, NK cells are also known to regulate the functions of other cells [1, 2]. Human NK cells are identified by the cell surface 
expression of CD16 and CD56. NK cells mediate their function through a number of important activating and inhibitory cell receptors listed in Table 1 [3]. It is thought that the balance between activating and inhibitory signals which NK cells receive from their surface receptors determines their functional fate. Many of the receptors listed in table 1 including CD16, killer immunoglobulin like receptors (KIR), NKG2 family of receptors which form a heterodimer with CD94, NKG2D and natural cytotoxicity receptors (NCR) have all been the subject of many studies. Likewise, several key cytokines, chemokines and adhesion molecules are found to have significant roles in maturation, differentiation, and effector function of NK cells. Much less is known about the function of Toll Like Receptors (TLRs), NOD-Like Receptors (NLRs) and RIG like Receptors (RLRs) in NK cell effector function.
Recent advances in our understanding of anti-tumor immune responses and cancer biology have revealed a complex dynamic interaction between the immune effectors and the tumor cells. Effectors of the immune system are known to shape the maturation of tumor cells, and to select for cancers with reduced immunogenicity. However, recent data from our laboratory indicated that the same effector mechanisms are likely responsible for shaping the maturation of healthy stem cells for the ultimate goal of the regeneration of damaged or disturbed tissues and the resolution of inflammation. Although, immunosuppression and tumor escape from immune recognition are thought to be major factors responsible for the establishment and progression of cancer, neither their underlying physiological significance nor the exact mechanisms by which immunosuppression occurs are completely understood.

Table I. List of NK cell Activating and Inhibitory surface receptors and their ligands.

\begin{tabular}{|l|l|}
\hline \multicolumn{1}{|c|}{ Receptors } & \multicolumn{1}{|c|}{ Ligands } \\
\hline Activating/inhibitory Receptors & \\
\hline FcyRIII (CD16) & Fc of antibodies \\
\hline CD2 & CD58(LFA-3) \\
\hline LFA-1 & ICAM-1 \\
\hline 2B4 & CD48 \\
\hline CD69 & Unknown \\
\hline DNAM-1 (CD226) & CD112, CD155 \\
\hline NKp80 & AICL \\
\hline Tactile (CD96) & CD155, CD111 \\
\hline TIGIT & CD112,CD113,CD155 \\
\hline CRTAM & TSLC1 \\
\hline C-type Lectin receptors -Activating/Inhibitory & \\
\hline CD94/NKG2A/B & HLA-E \\
\hline NKG2D & $\begin{array}{l}\text { MICA, MICB, ULBP-1, ULBP -2, } \\
\text { ULBP -3, ULBP -4, ULBP -5, ULBP -6 }\end{array}$ \\
\hline CD94/NKG2C & HLA-E \\
\hline CD94/NKG2E/H & HLA-E, Qa-1b \\
\hline Natural cytotoxicity receptors (NCR) & \\
\hline NKp46 (NCR1) & Viral Hemagglutinin \\
\hline NKp44 (NCR2) & Viral Hemagglutinin \\
\hline NKp30 (NCR3) & B7h6, HCMV-Pp65 \\
\hline Killer IG-like (KIR) - Activating/Inhibitory & HLA-C, HLA-B, HLA-A, HLA-G \\
\hline KIR2DLs, KIR3DLs, KIR2DS & $\begin{array}{l}\text { Cytokines, growth factors and } \\
\text { chemokine ligands }\end{array}$ \\
\hline Cytokines, growth factors and chemokines \\
\hline Toll-like receptors (TLR), NOD-like receptors & $\begin{array}{l}\text { Bacterial DNA, LPS, } \\
\text { peptidoglycan, teichoic acids, } \\
\text { flagellin, pilin, viral dsRNA and } \\
\text { fungi zymosan }\end{array}$ \\
\hline (NLR) and RIG-I-like receptors (RLR) & \\
\hline & \\
\hline
\end{tabular}


A number of factors responsible for the suppression of NK cell cytotoxicity in humans have been identified previously $[4-9,10]$. It is shown that freshly isolated tumor infiltrating NK cells are not cytotoxic to autologous tumors. Moreover, NK cells obtained from the peripheral blood of patients with cancer have significantly reduced cytotoxic activity [11-14]. In addition, NK cell cytotoxicity is suppressed after their interaction with stem cells [15-17]. In contrast, interaction of NK cells with the resistant tumors does not lead to a significant suppression of NK cell cytotoxicity when compared to those dissociated from the NK sensitive target cells $[18,19]$. Traditionally, the suppression of NK cell cytotoxic function after their interaction with the sensitive cells was sometimes perceived to be due to the exhaustion of cytotoxic granules from the NK cells, however, our recent data indicates that such suppression is physiological and it is an important step in maturation of NK cells to support differentiation of other cells, and in the resolution of inflammation.

Many mechanisms have been proposed for the functional inactivation of tumor associated NK cells including the over-expression of Fas ligand, the loss of mRNA for granzyme B [5] and decreased CD16 and its associated zeta chain [20], some of which are also observed in NK cells which have been conditioned to support differentiation of healthy stem cells (data not shown).

In this report we review the previous studies from our laboratory regarding the factors and mechanisms involved in NK cell immunosuppression observed in cancer, and after interaction with healthy stem cells, and furthermore we discuss the emerging view from our laboratory which indicates that NK cells are the effectors of selection, differentiation and resistance of undifferentiated or stem like cells. In this regard we have reported previously that the stage of maturation and differentiation of healthy untransformed stem cells such as hESCs and hiPSCs, hMSCs and hDPSCs as well as transformed tumorigenic cancer stem cells is predictive of their sensitivity to NK cell lysis [21]. Based on our results, we propose that NK cells play a significant role in differentiation of the cells by providing critical signals via secreted cytokines as well as direct cell-cell contact. To be conditioned to drive differentiation, NK cells may have to first receive signals through their key surface receptors either from healthy stem cells or those which have disturbed or defective capabilities to differentiate. In addition, NK cells by targeting other immune inflammatory cells or fibroblasts in the tumor microenvironment may become conditioned to lose cytotoxicity and gain cytokine producing phenotype be- fore they can aid in differentiation of stem cells. These alterations in NK cell effector function will ultimately aid in driving differentiation of a population of surviving healthy as well as transformed stem cells. In cancer patients since the majority of NK cells have lost cytotoxic activity, they may eventually contribute rather than halt the progression of cancer by allowing the growth and expansion of the pool of cancer stem cells. Below we will describe the evidence regarding the conditioning of the NK cells through key surface receptors and cytokines, differential targeting of stem cells and differentiated cells by the NK cells, and finally the role of conditioned NK cells in driving differentiation and resistance of undifferentiated tumors and healthy stem cells. In this regard conditioned NK cells function similarly to $\mathrm{T}$ regulatory cells in providing anti-inflammatory signals resulting in the resolution of inflammation and regeneration of the tissues.

\section{Induction of split anergy in NK cells after their co-culture with sensitive but not re- sistant tumors and after the triggering of CD16 and NKp46 but not NKp30 or NKp44 receptors}

We have previously shown that K562, an NK sensitive tumor, causes loss of NK cell cytotoxicity and induces cell death in a small subset of NK cells $[18,19]$. On the other hand, NK resistant tumors such as RAJI cells induce much less anergy or loss of NK cell cytotoxicity $[18,19]$. Furthermore, following NK cell cultures with sensitive tumor-target cells but not resistant tumors, the target binding NK cells undergo phenotypic and functional changes. Target cell inactivated NK cells express CD16-CD56dim/- CD69+ phenotype $[18,19]$. This phenotype has also been observed in several disease manifestations including HIV infection [22]. Significant down-modulation of CD16 receptor expression and decreased NK cell cytotoxic function were also seen in several cancer patients including those of the oral and ovarian cancer patients [23, 24]. In addition, down-regulation of CD16 surface receptors on NK cells was also observed when NK cells were treated with CA125 isolated from ovarian tumor cells [25]. The decrease in CD16 surface receptors was accompanied by a major decrease in NK cell killing activity against K562 tumor cells [25]. These observations suggested that CD16 receptors could likely play an important role in target cell induced loss of NK cell cytotoxicity. Indeed, CD16:Ig fusion proteins were shown to bind to a variety of tumor-target cells indicating the existence of specific ligands for CD16 receptors on tumor cells [26]. Fur- 
thermore, we have previously shown that the triggering of CD16 on untreated or IL-2 treated NK cells was found to result in down-modulation of CD16 receptors and in a great loss of cytotoxicity in NK cells. In addition, a subset of NK cells was programmed to undergo apoptosis [18, 19, 27, 28]. Cell death of NK cells was shown to be regulated, in part, by endogenously secreted TNF- $\alpha$ from the NK cells [28]. Previous studies by other groups have also shown that IL-2 activated NK cells undergo cell death following cross-linking of the CD16 receptor $[29,30]$. Thus, we have coined the term "split anergy" for the responses observed by NK cells after their interaction with sensitive target cells or after the triggering of CD16 receptors by the antibody in combination with IL-2 treatment [18, 19, 28, 31, 32]. Indeed, three subpopulations of NK cells; namely Free, Binder and Killer NK cells with varying degrees of loss of cytotoxicity were identified after the formation of conjugates with K562 targets [27, 33-36]. Free cells which did not bind or form conjugates with target cells were inactivated less, or exhibited the most cytotoxicity, whereas both Binder and Killer subsets exhibited significant loss of cytotoxicity. In contrast, Binder and Killer subsets but not Free NK subset secreted significant levels of cytokines and exhibited CD16-CD56dim/-CD69+ phenotype [27, 33-36]. Treatment of NK cells with IL-2 and anti-CD16mAb also induced split anergy by significantly decreasing the NK cell cytotoxicity while increasing the cytokine secretion capabilities of NK cells. Furthermore, NK cells exhibited CD16-CD56dim/-CD69+ phenotype after treatment with the combination of IL-2 and anti-CD16mAb $[28,31,32,37]$. Loss of cytotoxicity in NK cells was significantly exacerbated when NK cells were either treated with $\mathrm{F}(\mathrm{ab})^{\prime}{ }_{2}$ fragment of anti-CD16 $\mathrm{mAb}$ or treated with a combination of MHC-Class I and anti-CD16 mAbs while the same treatments resulted in an increased secretion of cytokines [32, 37]. Based on our recent results NKp46 mAb but not those of NKp30 or NKp44 were also able to induce significant NK cell anergy in the presence and absence of IL-2 correlating with their increased expression on untreated and IL-2 treated NK cells when compared to NKp30 and NKp44 expression (manuscript submitted). Moreover, addition of bacteria or their extracts conditioned NK cells to support differentiation of OSCSCs (unpublished results). These results suggested that receptor signaling in NK cells in the presence of IL-2 is likely to result in a decrease in NK cell cytotoxicity while increasing secretion of cytokines by the NK cells. Therefore, three distinct functional outcomes could be observed in NK cells which have either interacted with sensitive tumor-target cells or were treated with anti-CD16 or anti-NKp46 $\mathrm{mAbs}$ in the presence of IL-2 treatment to induce split anergy, namely; 1-loss of cytotoxicity, 2-gain in the ability to secrete cytokines and 3- death in a small subset of NK cells.

\section{Split anergy in NK cells is induced by total populations of monocytes and those de- pleted of CD16+ subset}

When hMSCs or hDPSCs were cultured with either viable or irradiated monocytes before they were exposed to IL2-treated NK cells a significant decrease in NK cell mediated cytotoxicity could be observed against hMSCs or hDPSCs. Interestingly, lysis of hMSCs and hDPSCs by untreated NK cells was also reproducibly blocked by the addition of monocytes [38]. To determine whether depletion of CD16+ subset of Monocytes from the total population of Monocytes change the cytotoxic function of NK cells in a 3 way interaction with the stem cells we used both the unsorted, and those sorted to remove CD16+ subsets in a 3 way killing assay with the NK cells. Both the total populations of monocytes and CD16- subsets were capable of inducing inhibition of NK cell cytotoxicity against stem cells [38]. We then determined whether decreased lysis of stem cells by NK cells was due to a competitive lysis of monocytes by the NK cells. We confirmed that monocytes were also lysed by the NK cells significantly. Furthermore, when we co-cultured stem cells with monocytes and sorted to remove the monocytes from the stem cells before assessing the killing function of NK cells, we could still observe significant inhibition of NK cell mediated lysis, arguing against the protection of stem cell lysis by NK cells being solely on the bases of competitive lysis of monocytes [38]. Therefore, even though lysis of monocytes by the NK cells may in part contribute to the prevention of NK cell lysis of stem cells, interaction of monocytes with stem cells can also provide resistance of stem cells against NK cell cytotoxicity. Decrease in NK cell lysis of hMSCs and hDPSCs was paralleled with a significant induction of IFN- $\gamma$. Indeed, when hMSCs or hDPSCs were cultured with IL-2 treated NK cells alone we could observe significant induction of IFN- $\gamma$ secretion. However, the highest increase was seen when IL2-treated NK cells were cultured with hMSCs or hDPSCs in the presence of monocytes. Therefore, although decreased killing of stem cells by the NK cells could be observed in the presence of monocytes, synergistic secretion of IFN- $\gamma$ by the NK cells in the presence of monocytes and stem cells could be observed, indicating an inverse relationship between cytotoxicity and IFN- $\gamma$ secretion 
(split anergy). This was similar to the profiles which we had seen when NK cells were treated with IL-2 and anti-CD16 $\mathrm{mAb}$ in which significant decrease in cytotoxicity of NK cells could be observed in parallel with increased secretion of IFN- $\gamma$ [28].

\section{Immunosuppressive effectors in tumor and non-transformed inflammatory microenvi- ronments}

Both the tumor microenvironment as well as non-transformed inflammatory microenvironment consists of a number of heterogeneous cell populations with ability to suppress and limit the function of cytotoxic immune effectors. Patients with cancer often have higher numbers of immature monocytes serving as Myeloid Derived Suppressor Cells (MDSCs) expressing CD14+HLADR- phenotype [39, 40]. Tumor associated Macrophages (TAMs) were previously shown to significantly influence and limit immune activation in the tumor microenvironment [41, 42]. In addition, MDSCs which are comprised of a number of distinct cell populations of myeloid origin and whose roles in immunosuppression have received significant attention in recent years are major cells capable of suppressing the cytotoxic function of $\mathrm{T}$ and $\mathrm{NK}$ cells [40]. T cell dysfunction is shown to be induced by MDSCs by the increased secretion of IL-10, TGF- $\beta$, induction of reactive oxygen species (ROS), and increased expression of arginase- 1 and inducible nitric oxide synthase (iNOS). T regulatory (Treg) and DC regulatory (DCreg) cells were also recently shown to have significant immunosuppressive roles in the tumor microenvironment [40]. Perhaps one of the most intriguing observations regarding the immunosuppressive effectors is the identification of Cancer Associated Fibroblasts (CAFs) and Mesenchymal Stem Cells (MSCs) as two potential tumor promoters. Fibroblasts from tumor tissues demonstrate an activated phenotype and have the ability to secrete many immunosuppressive factors such as TGF- $\beta$ and VEGF [43]. We have also found that undifferentiated fibroblasts, as well as MSCs and CD14+HLA-DR- monocytes are significantly more susceptible to NK cell mediated cytotoxicity [38], therefore, these cells may condition NK cells to undergo split anergy (please see below). Indeed, in oral epithelial tumors the majority of recruited immune effectors are usually found in the connective tissue area where through cell-cell interaction with the immunosuppressive cells such as fibroblasts, monocytes-macrophages and to a lesser extent $\mathrm{T}$ and $\mathrm{B}$ cells [38] can condition NK cells to lose cytotoxicity and gain in cytokine secretion capabilities, resulting in differentiation and resistance of oral epithelial tumors (please see below).

\section{Differentiated cells are lysed significantly less by the NK cells and do not trigger se- cretion of cytokines by the NK cells when compared to their undifferentiated coun- terparts}

Increased NK cell cytotoxicity and augmented secretion of IFN- $\gamma$ were observed when NK cells were co-incubated with OSCSCs which released significantly lower levels of GM-CSF, IL-6 and IL-8 and demonstrated decreased expression of phospho-Stat3, B7H1 and EGFR, and much lower constitutive NFKB activity when compared to differentiated OSCCs [21]. More importantly, OSCSCs expressed CD133 and CD44 $4^{\text {bright }}$ oral tumor stem cell markers [21]. Increase in IFN- $\gamma$ secretion was correlated with a decrease in secretion of IL-6 in co-cultures of NK cells with OSCSCs as compared to those co-cultured with OSCCs. Therefore, from these results a specific profile for differentiated NK resistant oral tumors emerged which demonstrated increased GM-CSF, IL-6 and IL-8 secretion in the context of decreased IFN- $\gamma$ secretion during their interaction with the NK cells. In contrast, co-cultures of cancer stem cells with NK cells demonstrated increased IFN- $\gamma$ in the context of lower GM-CSF, IL-6 and IL-8 secretion [21, 38]. In addition, three brain tumor stem cells which were previously characterized [44-46] were found to be significantly more susceptible to NK cell mediated cytotoxicity when compared to their differentiated counterparts which were significantly more resistant (manuscript submitted). Since OSCSCs and brain cancer stem cells were significantly more susceptible to NK cell mediated cytotoxicity we reasoned that healthy, non-transformed primary stem cells may also be susceptible to NK cell mediated cytotoxicity. We demonstrated previously that NK cells lysed hMSCs, hDPSCs and hESCs significantly. All different types of stem cells became resistant to NK cell mediated cytotoxicity once they were differentiated [21]. In addition, higher sensitivity of hiPSCs to NK cell mediated lysis was also observed when compared to parental line from which they were derived. Increased lysis of cancer stem cells or non-transformed healthy stem cells may be attributed to the use of allogeneic NK cells, however, our previous work using autologous NK cells exhibited similar levels of cytotoxicity against hDPSCs when compared to lysis by allogeneic NK cells [38]. Taken together these results indicated that undifferentiated cells were targets of both allogeneic and autologous NK cells. Thus, the stage of differentiation of the cells is predictive of their susceptibility to NK cell mediated cytotoxicity. 


\section{Blocking NFkB reverts the oral tumors to more of an undifferentiated phenotype re- sulting in an increase in NK cell mediated cytotoxicity.}

Since the degree of differentiation in the cells was predictive of their sensitivity to NK cell mediated cytotoxicity, we reasoned that blocking $\mathrm{NF \kappa B}$ in the cells may de-differentiate and subsequently revert the cells to more of undifferentiated phenotype, resulting in their increased susceptibility to NK cell mediated cytotoxicity. Indeed, blocking NFKB in oral tumors was found to increase CD44 surface receptor expression, which is one of the hallmarks of stem cells (unpublished results). In addition, we have shown previously that NFKB nuclear function in a primary Oral tumor OSCCs and in a non-tumorigenic oral cells (HOK-16B) as well as in an established tumor line, HEp-2 cells known to be Hela contaminant [47-50], modulates and shapes the function of interacting NK cells $[47,51]$. Since knock down of NFKB was shown to increase the function of immune inflammatory cells in diverse cell types (please see below) it is not surprising to find similar patterns of immune activation in both oral and non-oral derived tumor cells. In addition, since the majority, if not all cells, increase $\mathrm{NF \kappa B}$ during their activation and differentiation, a knock down of NFKB is likely to revert the cells, irrespective of their cellular origin, to a less differentiated phenotype resulting in the potential activation of the immune effectors in order to aid in their differentiation [21]. Similarly, inhibition of NFKB by Sulindac increased the functional activation of NK and enhanced anti-tumor cytotoxic activity $[47,51]$. Inverse modulation of IFN- $\gamma$ and IL- 6 cytokine secretion was seen in co-cultures of NK cells with NFkB knock down OSCCs, HOK-16B and HEp2 cells indicating that blocking NFKB in these cells serves to switch the balance from Th2 type responses to more of a Th1 type response [21, 51].

In agreement with our studies, targeted deletion of IKK- $\beta$ in epidermis of mice has previously been shown in one study to lead to inflammatory skin manifestations [52]. Elevated levels of cytokines and chemokines have also been demonstrated in the epi-

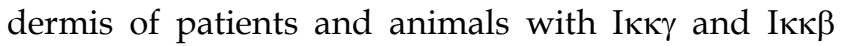
deletions [52, 53]. Mice with a keratinocyte-specific

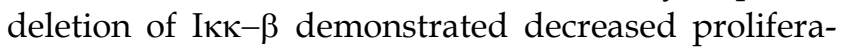
tion of epidermal cells and developed TNF- $\alpha$ dependent inflammatory skin disease [52]. In contrast, in other studies in which NFкB function was blocked in dermal keratinocytes by a mutant IкB- $\alpha$ an increased proliferation and hyperplasia [54] and eventual de- velopment of cutaneous squamous cell carcinomas of skin were seen if mice were allowed to survive and reach adulthood $[55,56]$. It is of interest to note that in these studies with diverse functional outcomes in keratinocytes, blocking TNF- $\alpha$ function resulted in the prevention of both the neoplastic transformation and the inflammatory skin disease $[52,56]$. Elevated numbers of immune inflammatory cells recruited to the site of epidermis are likely responsible for the increased secretion of TNF- $\alpha$. Indeed, we have demonstrated that synergistic induction of TNF- $\alpha$ could be observed when NFKB knock down oral tumors were cultured with either PBMCs or NK cells [51].

Since tumorigenic and non-tumorigenic human oral keratinocytes acquire sensitivity to NK cell mediated lysis when NFKB is inhibited, it is likely that this phenomenon is not specific to cancer or oral keratinocytes, and it may occur in other healthy non-transformed cell types. Indeed, when human primary monocytes were differentiated to dendritic cells they too became more resistant to NK cell mediated cytotoxicity [21]. Moreover, knock down of COX2 in primary mouse monocytes [21], or in mouse embryonic fibroblasts (unpublished observations), resulted in the reversion or de-differentiation of the monocytes and fibroblasts respectively, and the activation of NK cell cytotoxicity. Indeed, it is likely that any disturbance in cellular differentiation may predispose the cells to NK cell mediated cytotoxicity. Since STAT3 is an important factor increased during differentiation, blocking STAT3 is also critical in the activation of immune effectors [57]. In support of a critical role of STAT3 in immune evasion of tumor cells in humans, we and others have recently shown that glioblastoma multiforme (GBM) tumors display constitutive activation of STAT3 (Cacalano and Jewett, unpublished observation) [58], and poorly induce activating cytokines and tumor-specific cytotoxicity in human peripheral blood mononuclear cells (PBMCs) and NK cells. Ectopic expression of dominant-negative STAT3 in the GBM cells increased lysis of the tumor cells by the immune effectors and induced production of IFN- $\gamma$ by the interacting immune effectors (unpublished publications).

Since NFKB is shown to regulate IL-6 secretion in OSCCs, HOK-16B and HEp2 cells and secreted IL-6 in tumors is known to activate STAT3 expression and function, increase in NFKB nuclear function could in turn induce STAT3 activation and result in a significant resistance of tumors to NK cell mediated cytotoxicity. Therefore, targeted knock down of STAT3 or signaling pathways upstream of STAT3, such as $\mathrm{NF \kappa B}$, should de-differentiate the cells and predispose the cells to NK cell mediated cytotoxicity. 


\section{NK cells are conditioned in the tumor or in non-transformed inflammatory microenvi- ronment to drive differentiation}

The theory of immunosurveillance was postulated by Burnet [59] to indicate that the key thymus dependent effectors were responsible for clearance of tumors [60,61]. However, since then the concept of immunosurveillance has been expanded to include immunoediting as an important mechanism for the development of cancer $[62,63]$. It was suggested that cancer immunoediting comprises of three phases: elimination, equilibrium and escape [63]. Elimination represents the classical concept of immunosurveillance. However, during equilibrium and escape the interaction and cross signaling between the immune effectors, the tumor cells, and the effectors of the connective tissue in the tumor microenvironment is likely to result in the generation of differentiated tumors which are resistant to cytotoxic function of NK and CTLs and thus are capable of ceasing or lowering the inflammatory responses. Therefore, the final stages of cancer progression may result in the induction of less immunogenic tumors in the presence of fewer immune effectors capable of lysing them. Thus, pressures exerted by the tumor cells and immune effectors may eventually shape the microenvironment for the growth, expansion and invasion of tumors. Similarly, a variation of such interactions may also be observed during the interaction of NK cells with healthy non-transformed human stem cells in which case the three phases of interaction may include elimination which marks the decrease in the numbers of stem cells or other immune effectors in the inflammatory microenvironment, potentially resulting in the selection of stem cells by the NK cells, induction of tolerance or anergy which denotes the conditioning of NK cells by the stem cells and/or by the other effectors of microenvironment to lose cytotoxicity and support maturation and differentiation of remaining stem cells, and finally the resolution phase which denotes the elimination of anergized NK cells and generation of less immunogenic differentiated cells. Similarities and differences between these phases in cancer and during stem cell maturation will be discussed below.

\section{Potential role of anergized NK cells in dif- ferentiation and regeneration of tissues}

Induction of split anergy in NK cells could be an important conditioning step responsible for the repair of tissues during pathological processes irrespective of the type of pathology. In tumors since the generation and maintenance of cancer stem cells is higher, the majority, if not all of the NK cells, may be conditioned to support differentiation and repair of the tissues and as such the phenotype of NK cells in tumor microenvironment as well as in the peripheral blood may resemble that of the anergic NK cells, i.e., decreased NK cell cytotoxicity, acquisition of CD16-/dimCD56dim/+CD69+ phenotype and augmented ability to secrete inflammatory cytokines (Fig. $1)$. Of course, the degree of the loss of NK cell cytotoxicity may be directly proportional to the load of cancer stem cells. Therefore, our results suggest two very important functions for the NK cells. One function is to limit the number of stem cells and immune inflammatory cells by selecting those with a greater potential for differentiation for the repair of the tissues and second to support differentiation of the stem cells and subsequent regeneration of the tissues. To achieve these tasks NK cells have to acquire two different phenotypes and be conditioned to carry out both functions successfully. CD16+CD56dimCD69subsets of NK cells are cytotoxic and will mediate cytotoxicity depending on which cells they encounter first. In respect to the oral squamous cell carcinomas since the majority of immune effectors can be found at the connective tissue area the chances are that they may first encounter and interact with either the other immune inflammatory cells or the effectors of connective tissue such as fibroblasts. However, there is also the possibility that NK cells may first encounter the stem cells at the base of the epithelial layer, in which case by eliminating their bound stem cells, they too can become anergized (Fig. 1). Surprisingly, allogeneic CTLs were also found to target Glioblastoma stem-like cells and not their differentiated counterparts (unpublished observation). By eliminating a subset of stem cells or after their interaction with other immune inflammatory cells or effectors of connective tissue NK cells could then be in a position to support differentiation of selected population of stem cells since they will be conditioned to lose cytotoxicity, induce cytokine and growth factor secretion and gain the CD16-/dimCD56dim/+CD69+ phenotype (Fig. 1). It is interesting to note that all of the immune effectors isolated from the oral gingival tissues of healthy as well as diseased gingiva have CD69+ phenotype, with the exception that the numbers of immune effectors are much less in the healthy oral gingival tissues when compared to diseased tissues (Fig. 2). Therefore, our results suggest two very important functions for the NK cells. One function is to kill and the other function is to be conditioned to support differentiation for the repair and regeneration of the tissues. 


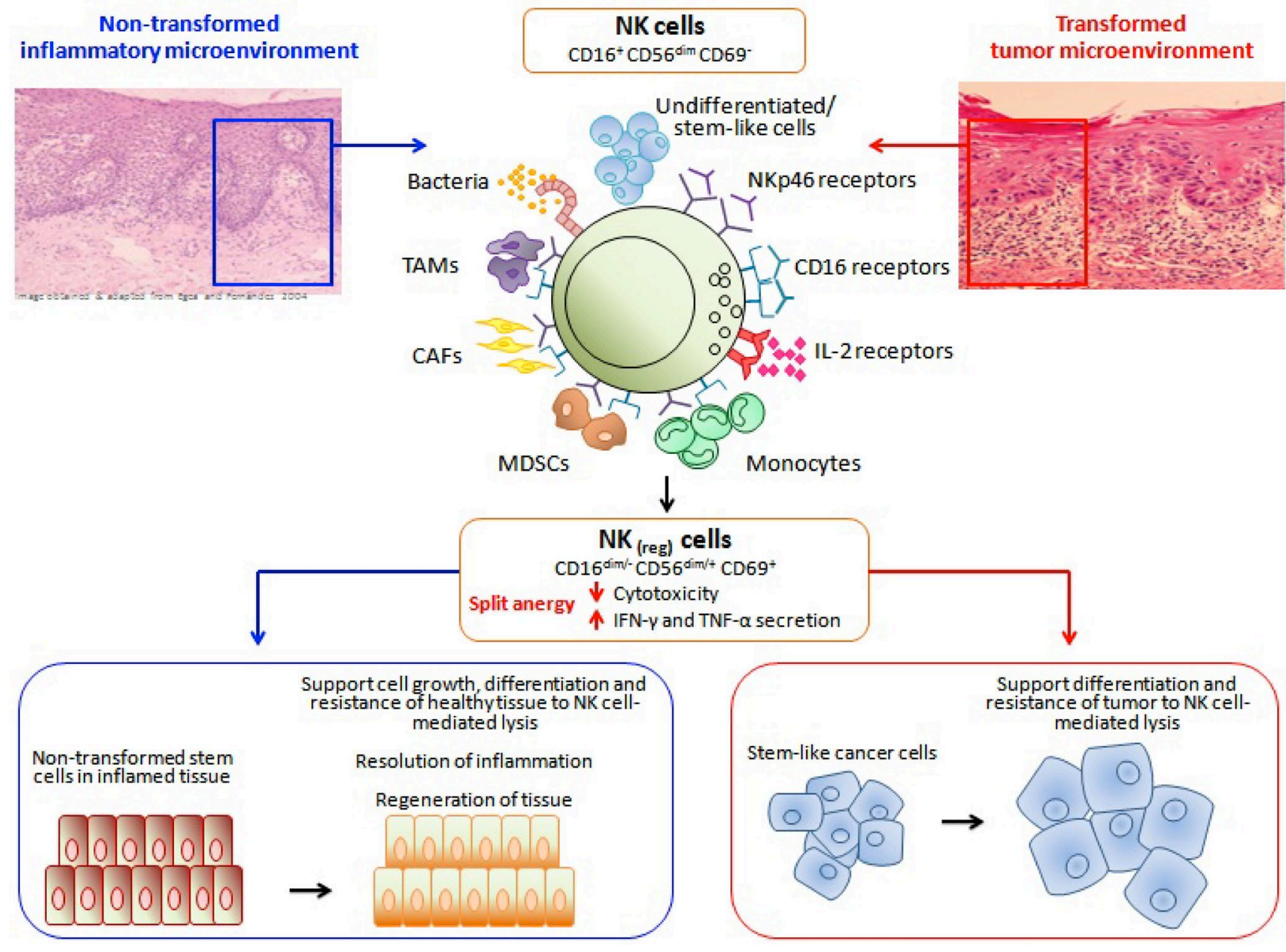

Fig. I. Hypothetical model of conditioning of NK cells by immune inflammatory cells and the effectors of connective tissue to modify NK cell phenotypic and functional properties in order to support differentiation of the cells and the resolution of inflammation. Hypothetical model of NK cell conditioning in the tumor microenvironment as well as in non-transformed immune inflammatory microenvironment is shown in this figure. Significant infiltration of immune effectors right beneath the epithelial layer can be seen in the connective tissue area where the immune inflammatory cells are likely to condition NK cells to lose cytotoxicity and gain the ability to secrete cytokines, a term which we have previously coined as split anergy in NK cells, and to support differentiation of the basal epithelial layer containing stem cells. NK cells are likely to encounter and interact with the other immune effectors such as monocytes or other myeloid-derived suppressor cells (MDSCs), and in tumor microenvironment with the tumor-associated macrophages

(TAMs), or with connective tissue-associated fibroblasts (CAF) in order to be conditioned to form regulatory NK cells (NK reg). NK cells may also directly interact with the stem cells at the base of the epithelial layer, in which case by eliminating their bound stem cells, they can become conditioned to support differentiation of other stem cells. In addition, bacteria through the binding to Toll like receptors can further aid in the generation of $\mathrm{NK}_{\text {reg. }}$. cells. All the above mentioned mechanisms may be operational during inflammatory processes in the tumor microenvironment or in healthy non-transformed inflammatory microenvironment. NK cell-differentiated epithelial cells will no longer be killed or induce cytokine secretion by the NK cells, therefore, resulting in the resolution of inflammation.

In vivo physiological relevance of above-mentioned observations could be seen in a subpopulation of NK cells in peripheral blood, uterine and liver NK cells which express low or no CD16 receptors, have decreased capacity to mediate cytotoxicity and is capable of secreting significant amounts of cytokines $[64,65]$. In addition, $70 \%$ of NK cells be- come CD16 dim or negative immediately after allogeneic or autologous bone marrow transplantation [64]. Since NK cells lose their cytotoxic function and gain in cytokine secretion phenotype and down modulate CD16 receptors after their interaction with tumor cells or the stem cells $[18,28]$, it is tempting to speculate that in vivo identified CD16- NK cells and 
in vitro tumor induced CD16- NK cells have similar maturational pathways since they have similar if not identical functional properties.

The proof of concept in support of this model was recently obtained in our laboratory. We observed that anergized NK cells were directly responsible for the increased differentiation and resistance of a number of different stem cells including cancer stem cells and dental pulp stem cells against cytotoxic effectors (manuscript submitted). In addition, we now have evidence which supports the notion that the induction of anergy in NK cells is an active process which is induced by the triggering of CD16 receptor on the NK cells and is not due to degranulation and exhaustion of cytotoxic granules (manuscript in prep).

Our work collectively suggests that anergized NK cells are as important as the non-anergized NK cells in their effector functions. NK cells are not only important for the removal and shaping of the size of the stem cells but also their differentiation, and the eventual regeneration of the new tissues. The task of NK cells in this regard goes above and beyond their most appreciated function of being the effectors of first line defense against viral infection and malignancies. They too are the effectors of differentiation and tissue regeneration.

\section{Tumor microenvironment may shape the function and phenotype of the NK cells}

The above observations prompted us to speculate regarding the significance of interaction of monocytes with NK cells and stem cells. It is plausible that monocytes may serve as shields against NK cell lysis of stem cells. Similar to anti-CD16 mAb mediated effect on IL2-treated NK cells, monocytes too can shield stem cells from killing by the NK cells by increasing the total IFN- $\gamma$ release by the NK cells while decreasing the cytotoxic function of NK cells (split anergy), resulting in an increased protection and differentiation of stem cells. Indeed, monocytes also increased TNF- $\alpha$, IL- 6 and VEGF secretion in the co-cultures of stem cells with NK cells which could augment NFKB and increase differentiation of stem cells. The shielding effect of monocytes could be a more generalized function of other effectors since NK cells can also target fibroblasts and to a much lesser extent the $\mathrm{T}$ and $\mathrm{B}$ cells [38]. Whether other MDSCs such as PMNs, mast cells, etc could also be targeted by the NK cells awaits future investigation. This may have significant implications for the role of NK cells in not only limiting inflammation, but also the significance of other immune effectors in shielding and limiting the cytotoxic function of NK cells against cancer or healthy stem cells in order to raise maximally the secretion of key cytokines for speedy and optimal differentiation of stem cells during inflammation. This is precisely what is observed in cancer patients in whom global decrease in NK, cytotoxic T cells and monocytes have all been reported [9].

\section{Potential homeostatic regulation of differ- entiation of stem cells in the tissue micro- environment by conditioned NK cells}

Recent data from our laboratory indicates that NK cells may be capable of regulating homeostatic differentiation of the stem cells. In this regard, the two stages of NK cells to mediate cytotoxicity and secretion of cytokines may be important for homeostatic regulation of cell differentiation since cytotoxic NK cells if they first encounter cancer stem cells or healthy stem cells they will likely eliminate them, however, if they encounter them after they are conditioned by the effectors of stroma, they may drive stem cell differentiation. Some of the terminally differentiated tumor cells or healthy cells may also be eliminated by cytokines secreted from the conditioned NK cells. Therefore, the potential outcome of such regulation by the NK cells may be the generation and maintenance of moderately to highly differentiated cells. These two stages of NK cell development may ensure that the number of stem cells as well as terminally differentiated cells remain very low in the stroma favoring the generation of moderately to highly differentiated cells. These hypotheses are currently under investigation in our laboratory. Indeed, as indicated above, all of the immune effectors dissociated from either healthy gingival tissues or periodontal gingiva are found to have an activated phenotype when compared to peripheral blood since they have elevated CD69 expression, and the magnitude of CD69 expression is similar between those recovered from healthy and periodontal gingivae, with the periodontal gingiva having higher numbers of immune effectors when compared to healthy gingiva (Fig. 2). Similarly, all $\mathrm{T}$ cells in normal breast tissue as well as breast tumor microenvironment were found to have high CD69 expression, with the breast tumor tissue having higher numbers of immune effectors when compared to normal breast tissue when compared to the peripheral blood [66]. The finding of CD69+ immune cells in healthy as well as tumor microenvironments suggest the general role of the activated immune effectors in repair and maintenance of tissue homeostasis, however, since potentially more cancer stem cell is generated by the tumor cells, immune cells may become overwhelmed and are unable to cope with the proliferating tumor cells. 

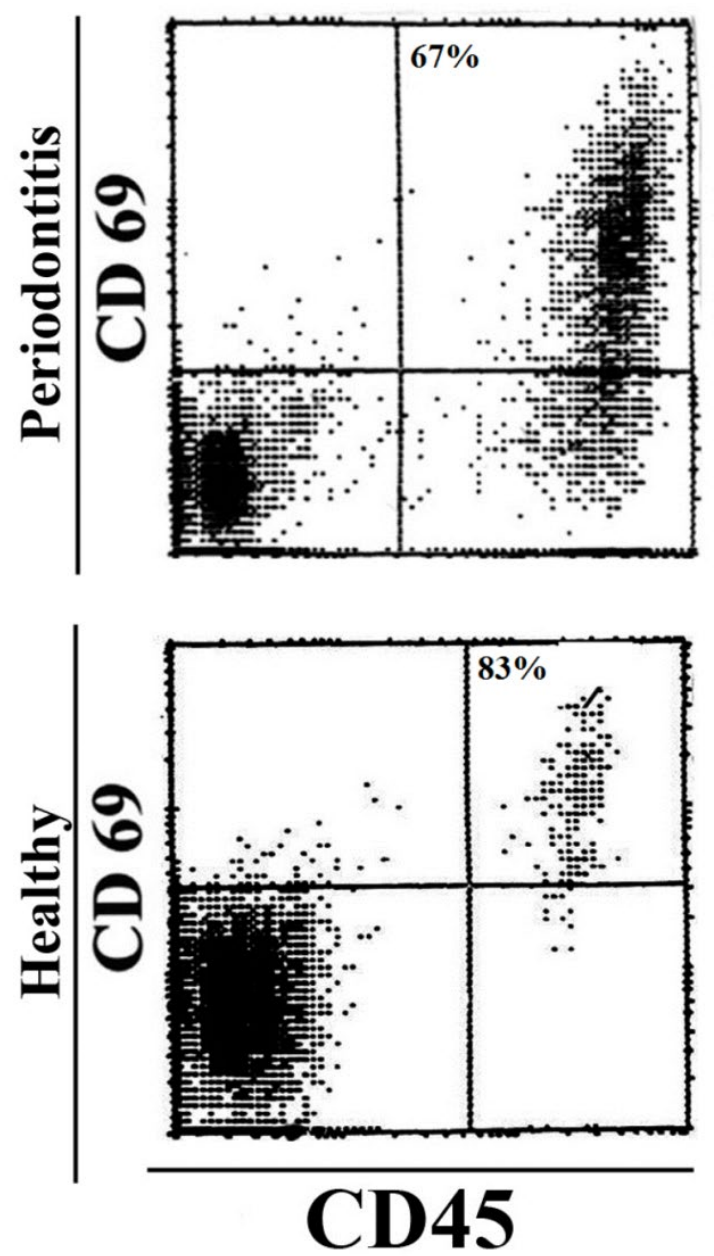

Fig. 2. The majority of the immune effectors in healthy and periodontal gingiva are of activated phenotype. Immune infiltrates from healthy and periodontal patient gingiva were dissociated, and the levels of CD69 expression on CD45+ immune effectors were determined using flow cytometry.

\section{Common functional outcomes between conditioned/anergized NK cells and T reg- ulatory cells}

Because of the ability to drive differentiation, conditioned NK cells may have the ability to halt inflammation since differentiated cells are no longer targeted by the NK cells and they do not induce cytokine secretion by the NK cells $[67,68]$. This function of NK cells may be similar to $T$ regulatory cells since they are inhibitory and are capable of decreasing the magnitude of inflammation in a number of previous studies. Thus in this respect anergized or conditioned NK cells may have regulatory or tolerizing function (NKreg). Therefore, although immunosuppression in the tumor microenvironment is not advantageous for the cancer patients, it is indeed, an important function which may not only stimulate differentiation, but it may also halt inflammation.

Since all of the immune effectors in the mucosal immune system including in the oral cavity are of activated phenotype, these cells, particularly NK cells are likely conditioned in the mucosa to support differentiation and resistance of the epithelial cells. Such environment is anti-inflammatory since the majority of immune cells is tolerant of ingested food particles since it contains many regulatory cells including $\mathrm{T}$ cells, Dendritic cells and conditioned NK cells. However, once the threshold which keeps the inflammation at bay is decreased in the mucosa due likely to disturbance in homeostatic differentiation of the cells, immune inflammatory effectors are recruited and activated which may result in tissue damage and establishment of chronic inflammation. Indeed, our preliminary in vitro and in vivo observations in humans indicated that the consumption of a newly developed food supplement with potent ability to condition NK cells to support differentiation of OSCSCs and hDPSCs (manuscript in prep.) was able to relieve chronic inflammation and pain, and resulted in the resolution of inflammatory mouth ulcers, decrease in the periodontal pocket depth and decrease swelling and edema in the oral mucosa. Furthermore, the number of neutrophils in the blood of a donor who had chronically decreased levels of neutrophils for over ten years rose to the normal levels after the consumption of the food supplement. Both the number and function of NK cells in the blood was substantially higher after the consumption of supplement (manuscript in prep).

\section{Autologoues and allogeneic NK cells drive differentiation of stem cells to a similar extent and result in the resistance of dif- ferentiated cells to NK cell mediated cyto- toxicity}

To determine whether there are differences between conditioned/anergized autologoues and allogeneic NK cells in driving differentiation of dental pulp stem cells we obtained dental pulp stem cells after extraction of molars and highly purified NK cells from the same individuals and compared their ability to differentiate hDPSCs to conditioned/anergized NK cells obtained from different donors. Both formaldehyde fixed conditioned NK cells and soluble factors secreted from conditioned autologous and allogeneic NK cells were able to drive differentiation of hDPSCs as determined by the surface expression of CD44, and resulted in the resistance of differentiated hDPSCs against cytotoxicity mediated by freshly isolated un- 
treated and IL-2 treated NK cells. Moreover, autologous and allogeneic NK cells did not secrete cytokines when cultured with differentiated hDPSCs (manuscript submitted). The ability of NK cells to differentiate hDPSCs and cause resistance of the differentiated hDPSCs to NK cell-mediated cytotoxicity was blocked when the combination of antibodies and not a single antibody to the key cytokines secreted by the NK cells was added, indicating the significance of synergistic interaction of the cytokines in driving stem cell differentiation (manuscript submitted). Several targets of synergistic modulation of stem cell differentiation by the NK cells are identified and their role in driving differentiation of the stem cells is currently under investigation in our laboratory.

\section{Conclusion}

Recent advances in our understanding of anti-tumor immune responses and cancer biology have revealed a complex dynamic interaction between the immune effectors and the tumor cells in the tumor microenvironment. Effectors of the immune system are known to shape the tumor cells and to select for cancers with reduced immunogenicity. However, the same effector mechanisms are likely responsible for shaping the maturation of healthy stem cells for the ultimate goal of the repair and the regeneration of damaged or disturbed tissues and the resolution of inflammation. Much work has been done to identify strategies by which tumor cells evade the function of immune system. Altered expression of MHC molecules which block recognition and activation of $\mathrm{T}$ and NK cells are examples of mechanisms by which tumor cells evade the function of immune system. In addition, tumor cells by releasing immunosuppressive factors such as Fas, VEGF, IL-6, IL-10, TNF- $\alpha$, GM-CSF and IL-1 $\beta$, induce T and NK cell apoptosis, block lymphocyte homing and activation, and dampen macrophage and dendritic cell function. However, the same effector functions are also important in tissue repair. Furthermore, progress has been made in identification of the upstream mechanisms which control the expression of immunosuppressive factors in tumor cells. Two key control elements, NFKB and STAT3 were identified and shown to coordinately regulate the production of multiple tumor-derived immunosuppressive molecules and play a pivotal role in tumor cell immune suppression. The potential for these two signaling modules to repress immune responses is underscored by the finding that the pathways interact and may even amplify each other.

Based on the accumulated work presented in this review, we suggest that NK cells may have two sig- nificant functions; one that relates to the removal and/or selection of stem cells or undifferentiated cells. Additionally, NK cells may lyse other effectors in the connective tissue area including immune effectors such as monocytes or other MDSCs as well as fibroblasts in order to not only decrease inflammation but also become conditioned to promote differentiation of stem cells and eventual regeneration of the tissues. The second important task for NK cells is to support differentiation and promote tissue regeneration after altering their phenotype to cytokine secreting cells (Fig. 1). This process will not only remove cells that are damaged and have flaws in the differentiation process, but also it will ensure the regeneration of tissues and the resolution of inflammation. Thus, any disturbance in the NK cell function or in the process of differentiation of stem cells may result in chronic inflammation, causing continual tissue damage and recruitment of immune effectors to aid in tissue regeneration.

The inability of patient NK cells to contain cancer stem cells potentially due to the flooding of NK cells by proliferating cancer stem cells and conversion of NK cells to cytokine secreting cells may likely be one mechanism by which cancer may progress and metastasize. Therefore, there should be two distinct strategies by the NK cells to eliminate tumors, one which targets stem cells and the other which targets differentiated cells. Since cancer stem cells were found to be more resistant to certain chemotherapeutic drugs but sensitive to NK cell mediated killing while differentiated oral tumors were more resistant to NK cell mediated killing but relatively more sensitive to chemotherapeutic drugs, combination therapy should in theory be effective in the elimination of both undifferentiated and differentiated tumors. In addition, since a great majority of patient NK cells have modified their phenotype to support differentiation of the cells, they may not be effective in eliminating the cancer stem cells. Therefore, these patients may benefit from repeated allogeneic NK cell transplantation for elimination of cancer stem cells. In this regard, depletion of immunosuppressive effectors in the tumor microenvironment, which condition NK cells to lose cytotoxicity, via radiation or chemotherapeutic drugs should in theory provide a better strategy for successful targeting of tumors by the NK cells.

\section{Acknowledgement}

This work was supported in part by NIH and UCLA Academic Senate and School of Dentistry seed grants. 


\section{Competing Interests}

The authors have declared that no competing interest exists.

\section{References}

1. Fildes JE, Yonan N, Leonard CT. Natural killer cells and lung transplantation, roles in rejection, infection, and tolerance. Transpl Immunol. 2008; 19: 1-11.

2. Farag SS, Caligiuri MA. Human natural killer cell development and biology. Blood Rev. 2006; 20: 123-37.

3. Pegram HJ, Andrews DM, Smyth MJ, Darcy PK, Kershaw MH. Activating and inhibitory receptors of natural killer cells. Immunol Cell Biol. 2011;89: 216-24.

4. Kolenko V, Wang Q, Riedy MC, O'Shea J, Ritz J, Cathcart MK, et al. Tumor-induced suppression of $\mathrm{T}$ lymphocyte proliferation coincides with inhibition of Jak3 expression and IL-2 receptor signaling: role of soluble products from human renal cell carcinomas. J Immunol. 1997; 159: 3057-67.

5. Mulder WM, Bloemena E, Stukart MJ, Kummer JA, Wagstaff J, Scheper RJ. T cell receptor-zeta and granzyme B expression in mononuclear cell infiltrates in normal colon mucosa and colon carcinoma. Gut. 1997; 40: 113-9.

6. Camp BJ, Dyhrman ST, Memoli VA, Mott LA, Barth RJ, Jr. In situ cytokine production by breast cancer tumor-infiltrating lymphocytes. Ann Surg Oncol. 1996; 3: 176-84.

7. Gimmi CD, Morrison BW, Mainprice BA, Gribben JG, Boussiotis VA, Freeman GJ, et al. Breast cancer-associated antigen, DF3/MUC1, induces apoptosis of activated human T cells. Nat Med. 1996; 2: 1367-70.

8. Bennett MW, O'Connell J, O'Sullivan GC, Brady C, Roche D, Collins JK, et al. The Fas counterattack in vivo: apoptotic depletion of tumor-infiltrating lymphocytes associated with Fas ligand expression by human esophageal carcinoma. J Immunol. 1998; 160: 5669-75.

9. Jewett A, Head C, Cacalano NA. Emerging mechanisms of immunosuppression in oral cancers. J Dent Res. 2006; 85: 1061-73.

10. Lindgren A, Yun CH, Sjoling A, Berggren C, Sun JB, Jonsson E, et al. Impaired IFN-gamma production after stimulation with bacterial components by natural killer cells from gastric cancer patients. Exp Cell Res. 2011;317: 849-58

11. Tanaka H, Nakao M, Shichijo S, Itoh K. Nonsteroidal anti-inflammatory drugs differentially regulate cytokine production in human lymphocytes: up-regulation of TNF, IFN-gamma and IL-2, in contrast to down-regulation of IL-6 production. Cytokine. 1995; 7: 372-9.

12. Miescher S, Stoeck M, Qiao L, Barras C, Barrelet L, von Fliedner V. Preferential clonogenic deficit of CD8-positive T-lymphocytes infiltrating human solid tumors. Cancer Res. 1988; 48: 6992-8.

13. Qin J, Han B, Pang J. [The relationship between TIL from human primary hepatic carcinoma and prognosis]. Zhonghua Yi Xue Za Zhi. 1997; 77: 167-70.

14. Han X, Papadopoulos AJ, Ruparelia V, Devaja O, Raju KS. Tumor lymphocytes in patients with advanced ovarian cancer: changes during in vitro culture and implications for immunotherapy. Gynecol Oncol. 1997; 65: 391-8.

15. Aggarwal S, Pittenger MF. Human mesenchymal stem cells modulate allogeneic immune cell responses. Blood. 2005; 105: 1815-22

16. Selmani Z, Naji A, Zidi I, Favier B, Gaiffe E, Obert L, et al. Human leukocyte antigen-G5 secretion by human mesenchymal stem cells is required to suppress $\mathrm{T}$ lymphocyte and natural killer function and to induce CD4+CD25highFOXP3+ regulatory T cells. Stem Cells. 2008; 26: 212-22.

17. Spaggiari GM, Capobianco A, Abdelrazik H, Becchetti F, Mingari MC, Moretta L. Mesenchymal stem cells inhibit natural killer-cell proliferation, cytotoxicity, and cytokine production: role of indoleamine 2,3-dioxygenase and prostaglandin E2. Blood. 2008; 111: 1327-33. doi:10.1182/blood-2007-02-074997.

18. Jewett A, Bonavida B. Target-induced inactivation and cell death by apoptosis in a subset of human NK cells. J Immunol. 1996; 156: 907-15.

19. Jewett A, Bonavida B. Target-induced anergy of natural killer cytotoxic function is restricted to the NK-target conjugate subset. Cell Immunol. 1995; 160: 91-7.

20. Nakagomi H, Petersson M, Magnusson I, Juhlin C, Matsuda M, Mellstedt $\mathrm{H}$, et al. Decreased expression of the signal-transducing zeta chains in tumor-infiltrating T-cells and NK cells of patients with colorectal carcinoma. Cancer Res. 1993; 53: 5610-2.
21. Tseng HC, Arasteh A, Paranjpe A, Teruel A, Yang W, Behel A, et al. Increased lysis of stem cells but not their differentiated cells by natural killer cells; de-differentiation or reprogramming activates NK cells. PLoS One. 2010;5: e11590.

22. Hu PF, Hultin LE, Hultin P, Hausner MA, Hirji K, Jewett A, et al. Natural killer cell immunodeficiency in HIV disease is manifest by profoundly decreased numbers of CD16+CD56+ cells and expansion of a population of CD16dimCD56- cells with low lytic activity. J Acquir Immune Defic Syndr Hum Retrovirol. 1995; 10: 331-40.

23. Lai P, Rabinowich H, Crowley-Nowick PA, Bell MC, Mantovani G, Whiteside TL. Alterations in expression and function of signal-transducing proteins in tumor-associated $\mathrm{T}$ and natural killer cells in patients with ovarian carcinoma. Clin Cancer Res. 1996; 2: 161-73.

24. Kuss I, Saito T, Johnson JT, Whiteside TL. Clinical significance of decreased zeta chain expression in peripheral blood lymphocytes of patients with head and neck cancer. Clin Cancer Res. 1999; 5: 329-34.

25. Patankar MS, Yu J, Morrison JC, Belisle JA, Lattanzio FA, Deng Y, et al. Potent suppression of natural killer cell response mediated by the ovarian tumor marker CA125. Gynecol Oncol. 2005.

26. Mandelboim O, Malik P, Davis DM, Jo CH, Boyson JE, Strominger JL. Human CD16 as a lysis receptor mediating direct natural killer cell cytotoxicity. Proc Natl Acad Sci U S A. 1999; 96: 5640-4.

27. Jewett A, Gan XH, Lebow LT, Bonavida B. Differential secretion of TNF-alpha and IFN-gamma by human peripheral blood-derived NK subsets and association with functional maturation. J Clin Immunol. 1996; 16: 46-54.

28. Jewett A, Cavalcanti M, Bonavida B. Pivotal role of endogenous TNF-alpha in the induction of functional inactivation and apoptosis in NK cells. J Immunol. 1997; 159: 4815-22.

29. Ortaldo JR, Mason AT, O'Shea JJ. Receptor-induced death in human natural killer cells: involvement of CD16. J Exp Med. 1995; 181: 339-44.

30. Azzoni L, Anegon I, Calabretta B, Perussia B. Ligand binding to FC gamma $\mathrm{R}$ induces c-myc-dependent apoptosis in IL-2-stimulated NK cells. J Immunol. 1995; 154: 491-9.

31. Jewett A, Cacalano NA, Head C, Teruel A. Coengagement of CD16 and CD94 receptors mediates secretion of chemokines and induces apoptotic death of naive natural killer cells. Clin Cancer Res. 2006; 12: 1994-2003.

32. Jewett A, Teruel A, Romero M, Head C, Cacalano N. Rapid and potent induction of cell death and loss of NK cell cytotoxicity against oral tumors by $\mathrm{F}\left(\mathrm{ab} \mathrm{b}^{\prime}\right) 2$ fragment of anti-CD16 antibody. Cancer Immunol Immunother. 2008; 57: 1053-66.

33. Bonavida B, Lebow LT, Jewett A. Natural killer cell subsets: maturation, differentiation and regulation. Nat Immun. 1993; 12: 194-208.

34. Bonavida B, Braquet M, Lebow LT, Jewett A. Qualitative and quantitative analysis of subpopulations of cytotoxic effector cells by flow cytometry. J Lipid Mediat Cell Signal. 1994; 9: 19-25.

35. Jewett A, Bonavida B. Activation of the human immature natural killer cell subset by IL-12 and its regulation by endogenous TNF-alpha and IFN-gamma secretion. Cell Immunol. 1994; 154: 273-86.

36. Jewett A, Bonavida B. Interferon-alpha activates cytotoxic function but inhibits interleukin-2-mediated proliferation and tumor necrosis factor-alpha secretion by immature human natural killer cells. J Clin Immunol. 1995; 15: 35-44.

37. Jewett A, Bonavida B. MHC-Class I antigens regulate both the function and the survival of human peripheral blood NK cells: role of endogenously secreted TNF-alpha. Clin Immunol. 2000; 96: 19-28.

38. Jewett A, Arasteh A, Tseng HC, Behel A, Arasteh H, Yang W, et al. Strategies to rescue mesenchymal stem cells (MSCs) and dental pulp stem cells (DPSCs) from NK cell mediated cytotoxicity. PLoS One. 2010;5: e9874.

39. Vuk-Pavlovic S. Rebuilding immunity in cancer patients. Blood Cells Mol Dis. 2008; 40: 94-100.

40. Greten TF, Manns MP, Korangy F. Myeloid derived suppressor cells in human diseases. Int Immunopharmacol. 2011;11: 802-6.

41. Coffelt SB, Hughes R, Lewis CE. Tumor-associated macrophages: effectors of angiogenesis and tumor progression. Biochim Biophys Acta. 2009; 1796: 11-8.

42. Mantovani A, Sica A. Macrophages, innate immunity and cancer: balance, tolerance, and diversity. Curr Opin Immunol. 2010;22: 231-7.

43. Yaguchi T, Sumimoto H, Kudo-Saito C, Tsukamoto N, Ueda R, Iwata-Kajihara $\mathrm{T}$, et al. The mechanisms of cancer immunoescape and development of overcoming strategies. Int J Hematol.2011; 93: 294-300.

44. Oka N, Soeda A, Inagaki A, Onodera M, Maruyama H, Hara A, et al. VEGF promotes tumorigenesis and angiogenesis of human glioblastoma stem cells. Biochem Biophys Res Commun. 2007; 360: 553-9.

45. Inagaki A, Soeda A, Oka N, Kitajima H, Nakagawa J, Motohashi T, et al. Long-term maintenance of brain tumor stem cell properties under at 
non-adherent and adherent culture conditions. Biochem Biophys Res Commun. 2007; 361: 586-92.

46. Soeda A, Inagaki A, Oka N, Ikegame Y, Aoki H, Yoshimura S, et al. Epidermal growth factor plays a crucial role in mitogenic regulation of human brain tumor stem cells. J Biol Chem. 2008; 283: 10958-66.

47. Jewett A, Wang MY, Teruel A, Poupak Z, Bostanian Z, Park NH. Cytokine dependent inverse regulation of CD54 (ICAM1) and major histocompatibility complex class I antigens by nuclear factor kappaB in HEp2 tumor cell line: effect on the function of natural killer cells. Hum Immunol. 2003; 64: 505-20.

48. Murakami J, Asaumi J, Kawai N, Tsujigiwa H, Yanagi Y, Nagatsuka H, et al. Effects of histone deacetylase inhibitor FR901228 on the expression level of telomerase reverse transcriptase in oral cancer. Cancer Chemother Pharmacol. 2005; 56: 22-8.

49. Murakami J, Asaumi J, Maki Y, Tsujigiwa H, Nagatsuka H, Kokeguchi S, et al. Influence of CpG island methylation status in O6-methylguanine-DNA methyltransferase expression of oral cancer cell lines. Oncol Rep. 2004; 12: 339-45.

50. Abdulkarim B, Sabri S, Deutsch E, Chagraoui H, Maggiorella L, Thierry $\mathrm{J}$, et al. Antiviral agent Cidofovir restores p53 function and enhances the radiosensitivity in HPV-associated cancers. Oncogene. 2002; 21: 2334-46.

51. Jewett A, Cacalano NA, Teruel A, Romero M, Rashedi M, Wang M, et al. Inhibition of nuclear factor kappa B (NFkappaB) activity in oral tumor cells prevents depletion of NK cells and increases their functional activation. Cancer Immunol Immunother. 2006; 55: 1052-63.

52. Pasparakis M, Courtois G, Hafner M, Schmidt-Supprian M, Nenci A, Toksoy A, et al. TNF-mediated inflammatory skin disease in mice with epidermis-specific deletion of IKK2. Nature. 2002; 417: 861-6.

53. Berlin AL, Paller AS, Chan LS. Incontinentia pigmenti: a review and update on the molecular basis of pathophysiology. J Am Acad Dermatol. 2002; 47: 169-87.

54. Seitz CS, Lin Q, Deng H, Khavari PA. Alterations in NF-kappaB function in transgenic epithelial tissue demonstrate a growth inhibitory role for NF-kappaB. Proc Natl Acad Sci U S A. 1998; 95: 2307-12.

55. van Hogerlinden M, Rozell BL, Ahrlund-Richter L, Toftgard R. Squamous cell carcinomas and increased apoptosis in skin with inhibited Rel/nuclear factor-kappaB signaling. Cancer Res. 1999; 59: 3299-303.

56. van Hogerlinden M, Rozell BL, Toftgard R, Sundberg JP. Characterization of the progressive skin disease and inflammatory cell infiltrate in mice with inhibited NF-kappaB signaling. J Invest Dermatol. 2004; 123: 101-8.

57. Wang T, Niu G, Kortylewski M, Burdelya L, Shain K, Zhang S, et al. Regulation of the innate and adaptive immune responses by Stat-3 signaling in tumor cells. Nat Med. 2004; 10: 48-54.

58. Rahaman SO, Harbor PC, Chernova O, Barnett GH, Vogelbaum MA, Haque SJ. Inhibition of constitutively active Stat3 suppresses proliferation and induces apoptosis in glioblastoma multiforme cells. Oncogene. 2002; 21: 8404-13.

59. Burnet FM. The concept of immunological surveillance. Prog Exp Tumor Res. 1970; 13: 1-27.

60. Burnet FM. Immunological surveillance in neoplasia. Transplant Rev. 1971; 7: 3-25.

61. Burnet FM. Implications of immunological surveillance for cancer therapy. Isr J Med Sci. 1971; 7: 9-16.

62. Dunn GP, Bruce AT, Ikeda H, Old LJ, Schreiber RD. Cancer immunoediting: from immunosurveillance to tumor escape. Nat Immunol. 2002; 3: 991-8.

63. Dunn GP, Old LJ, Schreiber RD. The immunobiology of cancer immunosurveillance and immunoediting. Immunity. 2004; 21: 137-48.

64. Cooper MA, Fehniger TA, Caligiuri MA. The biology of human natural killer-cell subsets. Trends Immunol. 2001; 22: 633-40.

65. Nemeth E, Baird AW, O'Farrelly C. Microanatomy of the liver immune system. Semin Immunopathol. 2009; 31: 333-43.

66. Ruffell B, Au A, Rugo HS, Esserman LJ, Hwang ES, Coussens LM. Leukocyte composition of human breast cancer. Proc Natl Acad Sci U S A. 2012;109: 2796-801.

67. Tseng HC, Arasteh A, Paranjpe A, Teruel A, Yang W, Behel A, et al. Increased lysis of stem cells but not their differentiated cells by natural killer cells; de-differentiation or reprogramming activates NK cells. PloS one. 2010; 5: e11590. doi:10.1371/journal.pone.0011590.

68. Jewett A, Arasteh A, Tseng HC, Behel A, Arasteh H, Yang W, et al. Strategies to rescue mesenchymal stem cells (MSCs) and dental pulp stem cells (DPSCs) from NK cell mediated cytotoxicity. PloS one. 2010; 5: e9874. doi:10.1371/journal.pone.0009874. 